\title{
Fibulin-3 promoter methylation alters the invasive behavior of non-small cell lung cancer cell lines via MMP-7 and MMP-2 regulation
}

\author{
EUN JIN KIM ${ }^{1}$, SO YONG LEE ${ }^{1}$, MI KYUNG WOO ${ }^{2}$, SOO IM CHOI ${ }^{1,3}$, TAE RIM KIM ${ }^{1}$, \\ MIN-JUNG KIM ${ }^{1}$, KUG CHAN KIM ${ }^{1}$, EUN WIE CHO ${ }^{2}$ and IN GYU KIM ${ }^{1,3}$
}

\begin{abstract}
${ }^{1}$ Department of Radiation Biology, Environmental Radiation Research Group, Korea Atomic Energy Research Institute, 1045 Daedeok-Daero, Yusong-gu, Daejeon 305-353; 2 Daejeon-KRIBB-FHCRC Research Collaboration Center, Korea Research Institute of Bioscience and Biotechnology (KRIBB), 125 Gwahangno, Yuseong-gu, Daejeon 305-806; ${ }^{3}$ Department of Radiation Biology, University of Science and Technology, Yusong-gu, Daejeon 305-600, Republic of Korea
\end{abstract}

Received July 11, 2011; Accepted August 22, 2011

DOI: $10.3892 /$ ijo.2011.1191

\begin{abstract}
Fibulin-3, an extracellular glycoprotein, has been suggested as having functions in tissue regeneration and organogenesis. However, its role in cancer remains unclear. We show here that fibulin-3 was silenced by hypermethylation of the promoter region in the relatively invasive A549 non-small cell lung cancer (NSCLC) cells compared with less invasive H460 NSCLC cells. Enforced expression of fibulin-3 in A549 cells down-regulated cellular MMP-7 and MMP-2, which was followed by inhibition of cell invasiveness. Conversely, suppression of fibulin-3 expression with siRNA in H460 cells showed the opposite effect. These results indicate that fibulin-3 is a negative regulator of invasiveness in NSCLC and further studies are needed for its therapeutic applications in treatment of NSCLC.
\end{abstract}

\section{Introduction}

Promoter hypermethylation leading to transcriptional silencing of tumour suppressor genes is a well-characterized epigenetic change in tumours $(1,2)$. More than 100 genes with tumourspecific hypermethylation of the promoter have been reported, including the RB gene, the cyclin-dependent kinase inhibitor

Correspondence to: Dr In Gyu Kim, Department of Radiation Biology, Environmental Radiation Research Group, Korea Atomic Energy Research Institute, 1045 Daedeok-Daero, Yusong-gu, Daejeon 305-353, Republic of Korea

E-mail: igkim@kaeri.re.kr

Dr Eun Wie Cho, Daejeon-KRIBB-FHCRC Research Collaboration Center, Korea Research Institute of Bioscience and Biotechnology (KRIBB), 125 Gwahangno, Yuseong-gu, Daejeon 305-806, Republic of Korea

E-mail: ewcho@kribb.re.kr

Key words: fibulin-3, methylation, tumour suppressor, MMP-7, invasion, lung cancer gene $C D K N 2 A$ (p16), the mismatch repair enzyme gene $M L H 1$ and the von Hippel Lindau tumour suppressor gene $V H L$ (3-5). In non-small cell lung cancer (NSCLC) as well as premalignant lung lesions, dozens of tumour-related genes have been reported that are frequently silenced through hypermethylation, such as $A P C, C D H 13, K L K 10$, and EFEMPI $(6,7)$. Among them, EFEMP1, which encodes fibulin-3, was repeatedly reported as being aberrantly methylated, which made it a representative marker for early detection and diagnosis of NSCLC (6-8).

The fibulins are a family of secreted glycoproteins which are characterized by repeated epidermal growth factor (EGF)-like domains and a unique C-terminal structure (9). Seven members of the fibulin family have been identified and considerable evidence focuses on their structural roles within the extracellular matrix (ECM), which are important for normal organogenesis, vasculogenesis and embryonic development $(9,10)$. Fibulins have also been shown to modulate cell morphology, growth, adhesion and motility, and the dysfunction of certain fibulins occurs in a range of human disorders. Recent studies show that fibulin-5 is associated with tumour suppression via regulation of vascular endothelial growth factor signalling, tumour angiogenesis, cell proliferation and invasive behaviour (11-14). Fibulin-3, which is homologous to fibulin-5, is also involved in regulation of tumour angiogenesis and cell growth (15).

In our studies on the invasiveness of NSCLC cells by comparing gene expression in highly aggressive A549 cells with other NSCLC cells, such as H460 cells, we identified that the expression of fibulin-3 is almost completely suppressed in A549 cells by the aberrant hypermethylation of promoter, whereas it is constitutively expressed in H460 cells. Here, we investigated the function of fibulin-3 related to the invasiveness of A549 cells and analyzed their molecular events.

\section{Materials and methods}

Cell culture. The NSCLC cell lines A549 and H460 were obtained from the Korea Cell Line Bank (Seoul, Korea) and grown in RPMI-1640 medium supplemented with $10 \%$ (v/v) 
fetal bovine serum (FBS; Invitrogen, Carlsbad, CA, USA) and $1 \%$ penicillin/streptomycin. Cells were incubated at $37^{\circ} \mathrm{C}$ in a humidified atmosphere with $5 \% \mathrm{CO}_{2}$.

For the preparation of fibulin-3-enriched conditioned media, H460 cells or fibulin-3-overexpressing A549 cells were plated in $100 \mathrm{~mm}$ culture dish at a density of $5 \times 10^{5}$ cells per plate and incubated with RPMI medium containing 10\% FBS for $48 \mathrm{~h}$. Supernatants were collected, filtered through $0.2-\mu \mathrm{m}$ filters and used at the indicated concentrations.

DNA extraction and bisulfite modification. Genomic DNA was extracted from A549 or H460 cells using DNeasy Tissue kit (Qiagen, Hercules, CA, USA) according to manufacturer's protocol. Genomic DNA (400 ng) from each sample was modified by sodium bisulfite using the EZ DNA Methylation-Gold kit (Zymo Research, Orange, CA, USA) according to the manufacturer's instructions as described before $(16,17)$.

Pyrosequencing. Pyrosequencing of bisulfite modified DNA was performed to validate methylation status of candidate loci. The primers for amplification and sequencing were: 5'-TGG ATT TTA TAG GAG TTG GTT AGA AGT-3' (forward), 5'-biotin-ACC RCA ACC CAA AAT ACC AAT-3' (reverse) and 5'-TTTATAGGAGTTGGTTAGAAG-3' (sequencing). These primers were designed by Pyrosequencing (PSQ) Assay Design Program (Biotage AB, Uppsala, Sweden) and PCR was carried out as described previously $(16,17)$. All primers were obtained from Bioneer (Daejeon, Korea). Pyrosequencing of PCR products was performed using PyroGold Reagent kit (Biotage $\mathrm{AB})$ according to the manufacturer's instruction. Four $\mathrm{CpG}$ sites were analyzed and the expected sequence was TTG GGA YGT TGA GTA GTT TTA GGG GAT YGT YGY GTT A (Y $=\mathrm{T}$ or $\mathrm{C}$ : methylated positions). The methylation percentage at individual $\mathrm{CpG}$ sites was then analyzed using Pyro Q-CpG software (Biotage $\mathrm{AB}$ ) and the methylation percentage of fibulin-3 was calculated by averaging the methylation percentages at four CpG sites.

Construction of the fibulin-3 overexpression vector and transfection. A 1482-bp insert of human fibulin-3 was amplified from poly(A) mRNA from $\mathrm{H} 460$ cells by reverse transcription (RT)-PCR using the following primers: HindIII (forward): 5'-ATA TAA GCT TAT GTT GAA AGC CC-3' and XhoI (reverse): 5'-ATA TCT CGA GCT AAA ATG AAA ATG GCC CC-3'. Fibulin-3 complementary DNA (cDNA) inserts were cloned into the mammalian expression vector pcDNA3.1 (Invitrogen), and the resultant expression vector (pcFBLN3) was transfected into A549 cells using Lipofectamine 2000 (Invitrogen).

Silencing-RNA(siRNA) targeting offibulin-3. A549 cells $\left(1 \times 10^{5}\right)$ were transfected with $50 \mathrm{nM}$ Stealth ${ }^{\mathrm{TM}}$ RNA targeting fibulin-3 (Invitrogen) or Stealth RNAi Negative Control Medium GC (Invitrogen) using Lipofectamine ${ }^{\mathrm{TM}}$ RNAi MAX reagent (Invitrogen). Cells were incubated for $72 \mathrm{~h}$ after transfection at which time cells were harvested for RT-PCR or Western blot analyses. The sequences of Stealth RNA for targeting the fibulin-3 gene were as follows: sense, 5'-UAG AAU GUA GGG AUC UUG ACA AGG -3'; antisense, 5'-CCU UGU CAA GAU CCC UAC AUU CUA A-3'.
Table I. Primer sequences for RT-PCR.

Primer Sequences

Fibulin-1

Forward 5'-TGCTTCGTGGGCTACCAGCTGCTGT-3'

Reverse 5'-CTCCTCGTTGAGATGGTAGCCACGG-3'

Fibulin-2

Forward 5'-GAGATCCCTGAGAGTGGCACTGAGG-3'

Reverse 5'-GAGAAGGCACTCATCCTGGTCATCG-3'

Fibulin-3

Forward 5'-ATGTTGAAAGCCCTTTTCC-3'

Reverse 5'-CTAAAATGAAAATGGCCCC-3'

Fibulin-5

Forward 5'-ATGCCCAGGAATAAAAAGGATACTCAC-3'

Reverse 5'-TCAGAATGGGTACTGCGACACATATAT-3'

MMP-2

Forward 5'-ATGGAAGGCGCTAATGGCCCGGGGCG-3'

Reverse 5'-TCAGCAGCCTAGCCAGTCGGATTTG-3'

MMP-7

Forward 5'-ATGCGACTCACCGTGCTGTG-3'

Reverse 5'-CTATTTCTTTCTTGAATTAC-3'

MMP-9

Forward 5'-ATGAGCCTCTGGCAGCCCCTGGTCC-3'

Reverse 5'-CTAG TCCTCAGGGCACTGCAGGATG-3

GAPDH

Forward 5'-ATGGGGAAG GTGAAGG-3'

Reverse 5'-TTACTCCTTGGAGGCC-3'

cDNA synthesis and PCR amplification. Total RNA was isolated from H460 and A549 cells with TRIzol reagent (Invitrogen) following the manufacturer's instructions. First-strand cDNA was generated from $1 \mu \mathrm{g}$ of total RNA using oligo dT primers and a cDNA synthesis kit (Intron Biotechnology, Gyungki-do, Korea). Resultant cDNA served as templates for PCR amplification with primers shown in Table I. The PCR conditions were initial denaturation at $94^{\circ} \mathrm{C}$ for $5 \mathrm{~min}, 30$ cycles of $94^{\circ} \mathrm{C}$ for $1 \mathrm{~min}$, $56^{\circ} \mathrm{C}$ for $1 \mathrm{~min}$, and $72^{\circ} \mathrm{C}$ for $90 \mathrm{sec}$, and a final extension at $72^{\circ} \mathrm{C}$ for $10 \mathrm{~min}$. The amplified PCR products were analyzed on $1 \%$ agarose gels (Intron Biotechnology) with ethidium bromide.

Western blot analysis. Anti-MMP-2, anti-MMP-7, anti-MMP-9 and anti-fibulin-3 antibodies for Western blot analysis were purchased from Santa Cruz Biotechnology (Santa Cruz, CA, USA). Anti-phospho-ERK, anti-phospho-Akt, anti-phospho$\mathrm{NF}-\kappa \mathrm{B}$ (p65) and anti- $\beta$-actin antibodies were purchased from Cell Signaling (Beverly, MA, USA). Western blot analysis was performed as described previously (17).

Total cell lysates (30 $\mu \mathrm{g} /$ lane), which were prepared with RIPA buffer, were separated on a $10 \%$ or $15 \%$ sodium dodecyl sulfate-polyacrylamide gel and transferred to a nitrocellulose membrane (Hybond; Amersham Pharmacia, USA). The blots were blocked for $1 \mathrm{~h}$ at room temperature with blocking buffer 


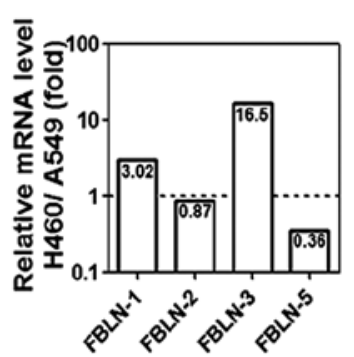

B

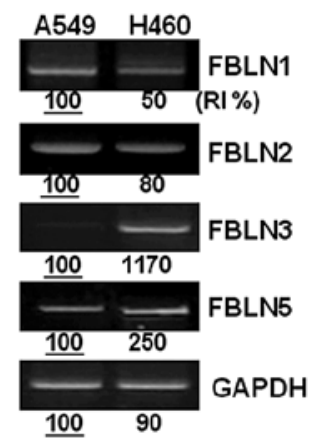

D
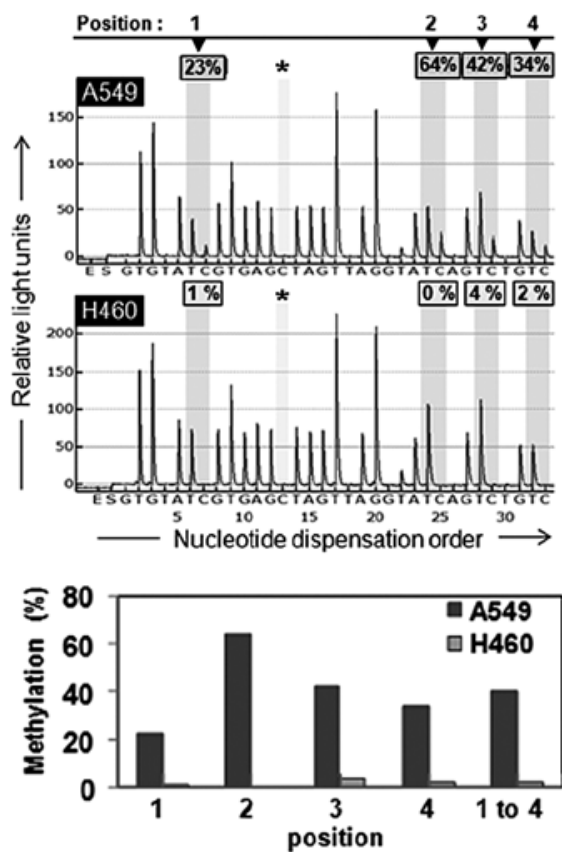

FBLN3 forward primer : 5 ' - TGGATTTTATAGGAGTTGGTTAGAAGT - 3 ' *FBLN3 reverse primer : 5 '(biotin)- ACCRCAACCCAAAATACCAAT- $3^{\prime}$ *FBLN3 sequencing primer : 5' - TTTATAGGAGTTGGTTAGAAG -3' *FBLN3 sequence to analyze : TTGGGAYGTTGAGTAGTTTTAGGGGATYGTYGYGTTA \& $\underline{Y}$ : methylated position 1 to 4

$*$ Number, +1 , indicates the translation start site of the FBLN3 gene.

Figure 1. Methylation of four CpG sites in the promoter region of fibulin-3. (A) Relative expression levels of genes of the fibulin family in H460 cells determined by microarray analysis compared with A549 cells. (B) RT-PCR analysis of expression of fibulin family genes in A549 and H460 cells. GAPDH was used as internal control. Band intensity was quantified using Iamge J software and relative intensity (RI \%) was calculated using the band intensity in A549 cells as reference value. (C) Original sequence of fibulin-3 and bisulphite-converted sequences. Each Y of underlined sequences indicates a methylated position. (D) Pyrosequencing diagram of A549 and $\mathrm{H} 460$ cells and the comparison of methylation percentages at each CpG position. Each gray colored box in the diagram indicates the position of the four Y's shown in panel C. The columns marked with * are the built-in quality control sites consisting of cytosines converted to thyamines. The last set of bars in the methylation percentage graph indicates the means of the methylation in positions 1-4.

(10\% non-fat milk in phosphate-buffered saline PBS containing $0.1 \%$ Tween 20). The membrane was incubated overnight in a cold chamber with specific antibodies. After being washed with TBS, the membrane was incubated with a horseradish peroxidase-labeled secondary antibody and visualized with the Westzol enhanced chemiluminescence detection kit (Intron Biotechnology). Protein concentration was determined with a Lowry kit (Bio-Rad, Hercules, CA, USA). The intensity of bands was evaluated using Image J software (http://www.imagej.nih. gov/ij).

Colony-forming assay. For the colony-forming assay, cells were plated in $35-\mathrm{mm}$ culture dishes at a density of $2 \times 10^{3}$ cells per plate after being transfected with fibulin-3-specific siRNA or fibulin-3-overexpression vector pcFBLN3. Cells were incubated for $10 \mathrm{~d}$ post-transfection and stained with $0.5 \%$ crystal violet. Colonies, defined as groups of $\geq 50$ cells, were then counted.

Invasion assay. Cell invasion was determined using Matrigelcoated invasion chambers $(8 \mu \mathrm{m}$ pores; BD Biosciences, Bedford, MA, USA) according to the manufacturer's instructions. A549 and $\mathrm{H} 460$ cells or cells at $48 \mathrm{~h}$ post-transfection with pcFBLN3 or fibulin-3-specific siRNA were incubated for $24 \mathrm{~h}$ in serum-free RPMI medium and then detached from the cell culture plates using a non-enzymatic cell dissociation solution (Sigma, St. Louis, MO, USA). Cells were resuspended in serumfree RPMI medium and placed in the upper invasion chamber ( $5 \times 10^{4}$ cells/well), and RPMI medium containing $10 \%$ (v/v) FBS was added to the lower chamber. The plates were incubated in a humidified $\mathrm{CO}_{2}$ atmosphere for $24 \mathrm{~h}$, and non-invasive cells in the upper chamber were removed by wiping with a cotton swab. The invasive cells in the lower invasion chambers were fixed with $4 \%(\mathrm{w} / \mathrm{v})$ formaldehyde in phosphate-buffered saline and stained with $2 \%(\mathrm{w} / \mathrm{v})$ crystal violet in $2 \%(\mathrm{v} / \mathrm{v})$ ethanol. The stained cells that penetrated through the Matrigel were counted under a light microscope.

\section{Results}

Methylation of the fibulin-3 promoter region suppressed fibulin-3 expression in A549 cells. A549 lung adenocarcinoma cancer cells are more resistant to cytotoxic agents and more invasive than H460 NSCLC cell line $(18,19)$. To identify novel genes involved in lung tumour metastasis, we compared the expression of 30,000 human genes between A549 and H460 cells through DNA microarray analyses (data not shown). Among hundreds of differentially regulated genes, fibulin-3 was prominent, of which the expression in $\mathrm{H} 460$ cells was about 16 times greater than in A549 cells. The low-level expression of the fibulin proteins in NSCLC cells compared with normal subjects was previously reported (6), but their exact roles relating to tumourigenesis or invasiveness remained unclear. We first evaluated the expression levels of the fibulin family proteins in A549 and H460 cells by comparing DNA microarray data and confirmed them by RT-PCR. As shown in Fig. 1A and B, the expression levels of fibulin-1, -2 and -5 in A549 cells were not significantly different 
A

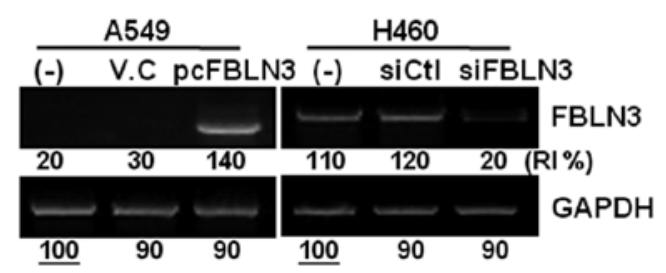

B

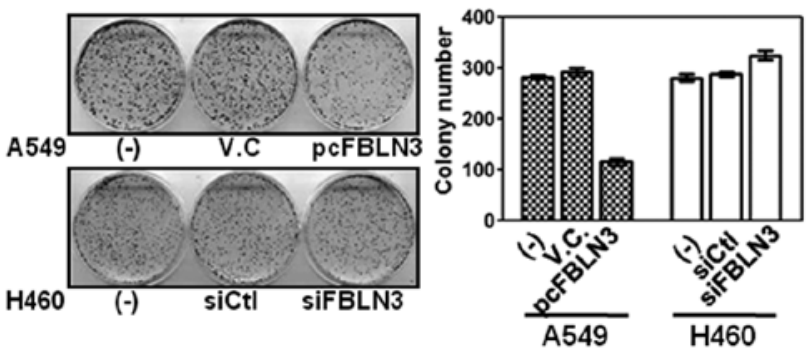

C
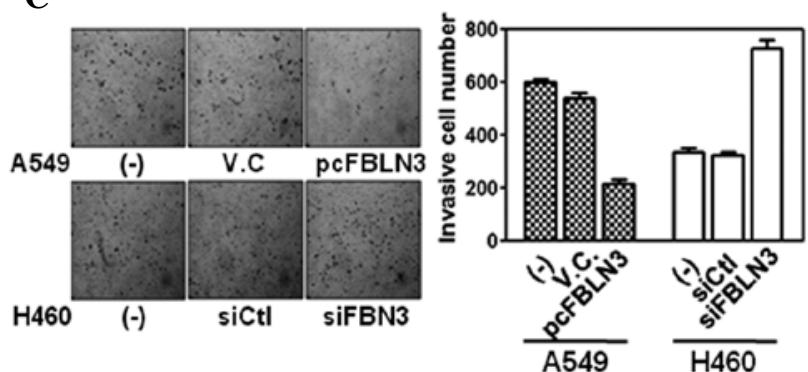

Figure 2. Fibulin-3 gene expression is associated with cell growth and invasive behaviour of lung cancer cells. (A) RT-PCR analysis of fibulin-3 expression in fibulin-3-overexpressing A549 cells and fibulin-3-suppressed H460 cells. RNA was extracted from cells at $72 \mathrm{~h}$ post-transfection. Relative intensity (RI \%) was calculated using the band intensity of GAPDH in untransfected cells as reference value. (B) Colony-forming assay of fibulin-3-overexpressed or fibulin3-suppressed NSCLC cell lines. (C) Matrigel assay of fibulin-3-overexpressed or fibulin-3-suppressed NSCLC cell lines. These assays were performed in triplicate, and representative data are shown.

from those of $\mathrm{H} 460$ cells; however, the expression of fibulin-3 was much higher (more than 10-fold) in H460 cells than in A549 cells.

To examine the involvement of epigenetic modifications on low-level expression of fibulin-3 in A549 cells, we analyzed the methylation patterns of the fibulin-3 gene promoter region in A549 and $\mathrm{H} 460$ cells by pyrosequencing. To predict putative CpG islands of fibulin-3, the promoter and 5'-untranslated region (5'-UTR) of fibulin-3 were analyzed using the Methprimer program (http://www.urogene.org //methprimer) (20), which showed three $\mathrm{CpG}$ islands. Among them, the second $\mathrm{CpG}$ island in 5'-UTR region corresponding to -1168 -1107 nucleotide of fibulin-3 was analyzed to validate methylation status and four CpG sites (position 1-4) were identified as hypermethylation sites (Fig. 1C). The average methylation percentage of these CpG sites was $40.8 \pm 17.52$ (mean $\pm \mathrm{SD}$ ) in A549 cells, whereas the methylation percentage of these positions in $\mathrm{H} 460$ cells was insignificant $(<2.0 \%)$. On the contrary, the first $\mathrm{CpG}$ island was not hypermethylated in A549 or $\mathrm{H} 460$ cells, which indicates that silencing of fibulin-3 in A549 cells is mediated by hypermethylation of the second $\mathrm{CpG}$ island, which functions as a methylated CpG hot spot.

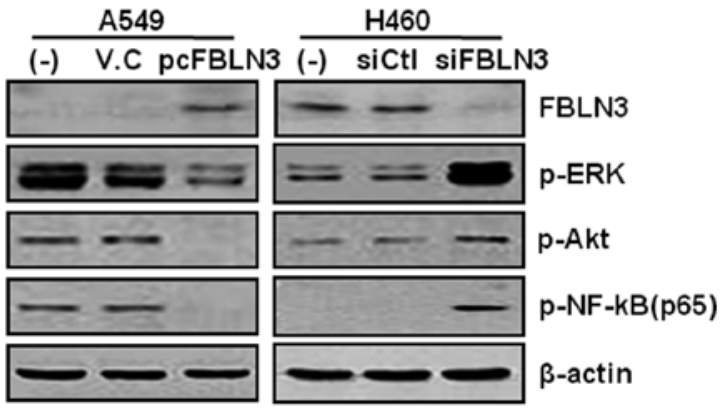

Figure 3. Western blot analysis of Erk, Akt and NF- $\kappa$ B signalling pathways, which are related to cell growth and metastasis in NSCLC cell lines. Proteins were extracted from cells at $72 \mathrm{~h}$ post-transfection, and $\beta$-actin was used as the control.

Fibulin-3 gene expression is associated with different invasive behaviour in $A 549$ and $H 460$ cells. To investigate whether the fibulin-3 gene is involved in regulation of cell growth and invasion in NSCLC, fibulin-3 was overexpressed in A549 cells by transfection of pcDNA/FBLN3, and they were analyzed by colony-forming or Matrigel motility assays (Fig. 2A). Additionally, the same assays were performed with $\mathrm{H} 460$ cells following suppression of fibulin-3 expression using fibulin3-specific siRNA (siFBLN3) (Fig. 2A). As shown by the colony-forming assay in Fig. 2B, overexpression of fibulin-3 significantly inhibited cell growth of A549 lung cancer cells, whereas suppression of fibulin-3 enhanced cell growth in H460 cells. The invasiveness of lung cancer cells was also influenced by fibulin-3 expression. As shown in Fig. 2C, overexpression of the fibulin-3 gene significantly inhibited invasion of A549 cells into the Matrigel, and suppression of fibulin-3 expression resulted in the opposite effect in $\mathrm{H} 460$ cells. These results indicate that the more invasive behaviour of A549 adenocarcinoma cells may be partially due to down-regulated fibulin-3 expression following increased methylation of the promoter region in A549 cells compared with $\mathrm{H} 460$ cells.

Fibulin-3 inhibits signalling pathways involved in cell growth and metastasis. The colony-forming assay depicted in Fig. 2A indicated that fibulin-3 levels can influence cell growth. Therefore, we examined the activation states of various signalling molecules related to cell growth, including ERK, AKT and $\mathrm{NF}-\kappa \mathrm{B}$. Activation of these signalling pathways is also involved in metastasis (21-24). As expected, these signalling molecules were seemed to be more activated by phosphorylation in A549 cells than in H460 cells (Fig. 3). By overexpression of fibulin-3 in A549 cells the phosphorylation of these molecules were severely inhibited. Conversely, suppression of fibulin-3 in H460 cells increased their phosphorylational activation (Fig. 3).

Fibulin-3 regulates the expression of MMP-7 and MMP-2. The matrix metalloproteinases (MMPs) are a family of proteinases associated with tumourigenesis with roles in ECM turnover and cancer cell invasion. MMPs can also alter cell growth by regulating signalling pathways (25). Therefore, we investigated MMPs which would be involved in fibulin-3 regulation of cancer cell invasiveness and growth. The expression levels of MMP-2, MMP-7 and MMP-9 were analyzed by RT-PCR and Western 
A

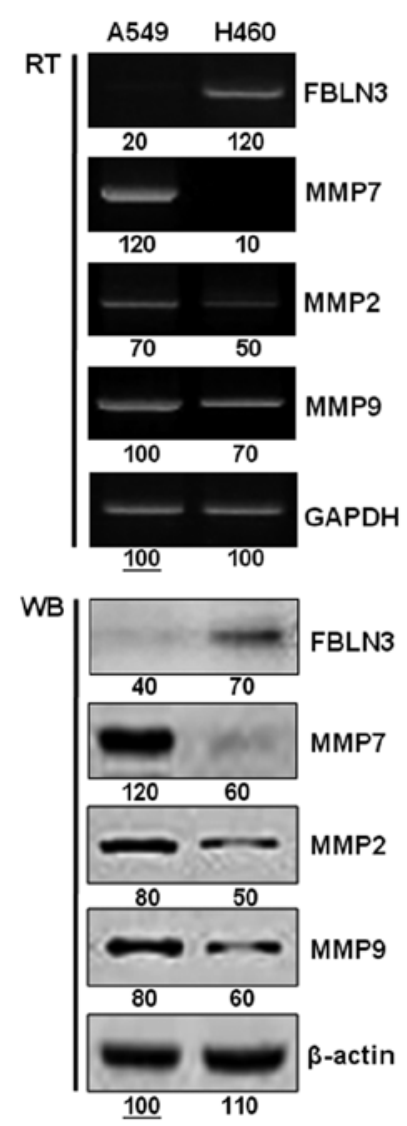

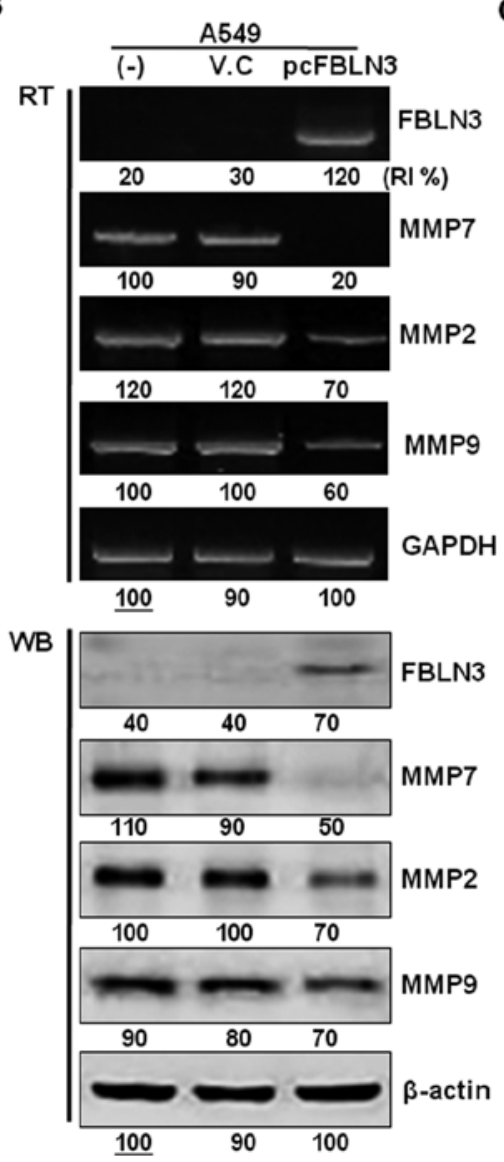

C

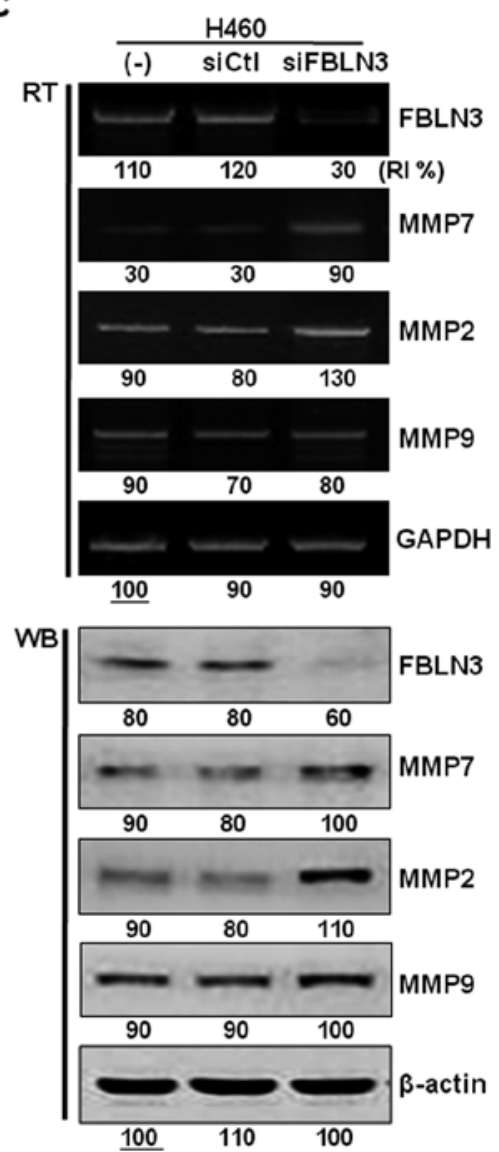

Figure 4. RT-PCR and Western blot analysis of MMP expression in A549 and H460 cells. (A) RT-PCR and Western blot analysis of MMPs in A549 and H460 cells. (B) RT-PCR and Western blot analysis of MMPs in fibulin-3-overexpressing cells transfected with the pcFBLN3 expression to transfection with fibulin-3-specific siRNA. Relative intensity (RI \%) of each band was calculated using the band intensity of GAPDH or $\beta$-actin in A549 or untransfected cells as reference value.

blotting. As shown in Fig. 4A, the expression of MMP-7 differed significantly between A549 and H460 cells. MMP-7 was much more abundant in A549 cells than in $\mathrm{H} 460$ cells, which expressed lower levels of fibulin-3. MMP-2 was also slightly more abundant in A549 cells. However, MMP-9 levels were not significantly different between the two cell lines.

The effects of fibulin-3 expression on MMP-7, MMP-2 and MMP-9 expression were further confirmed by overexpression of fibulin-3 in A549 cells or fibulin-3 suppression in H460 cells. MMP-7 expression was abolished in fibulin-3-overexpressing A549 cells, and MMP-2 expression was also diminished (Fig. 4B). Conversely, suppression of fibulin-3 significantly elevated cellular levels of MMP-7 and MMP-2 in H460 cells (Fig. 4C). Taken together, we concluded that the invasive behaviour of A549 cells induced by fibulin-3 suppression is mediated mainly by increased expression of MMP-7 and MMP-2.

Extracellular fibulin-3 regulates the expression of MMP-7 and $M M P-2$. Fibulin-3 is a secreted glycoprotein, which functions in extracellular space. To confirm the effect of fibulin-3 as an extracellular protein, A549 cells were treated with fibulin-3enriched conditioned media from fibulin-3-overexpressing cells (H460 or fibulin-3-overexpressing A549 cells) and their effects on cell growth and invasiveness were analyzed. As shown in Fig. 5A, the colony-forming ability of A549 cells was signifi- cantly decreased by increasing the concentration of conditioned media containing fibulin-3 (Fig. 5A). When cell culture media of A549 cells were completely exchanged to fibulin-3-enriched conditioned media, no colonies were formed. Cell morphology was also changed to elongated form by adding fibulin-3 enriched media (data not shown). More importantly the expression of MMP-2 and -7 were decreased severely, which might mean the decrease of invasiveness of A549 cells (Fig. 5B). Hundreds of functional proteins which can modulate cells, including fibulin-3, might exist in conditioned media, although the conditioned media from fibulin-3-overexpressing A549 cells and H460 cells exerted the same effect on A549 cells, which implicates the action of fibulin-3. To confirm the effect of fibulin-3 on A549 cells, anti-fibulin-3 antibody was added to the conditioned media and the effect of blocking fibulin-3 was examined. As shown in Fig. 5C, the effects of conditioned media containing fibulin-3 were completely reversed by the presence of the specific antibody against fibulin-3, confirming its function as a modulator of invasiveness of NSCLC cells.

\section{Discussion}

Fibulin-3, which is also known as EFEMP1 (EGF-containing fibulin-like extracellular matrix protein 1) or FBNL (fibrillinlike), is a member of the fibulin family of ECM proteins that 
A
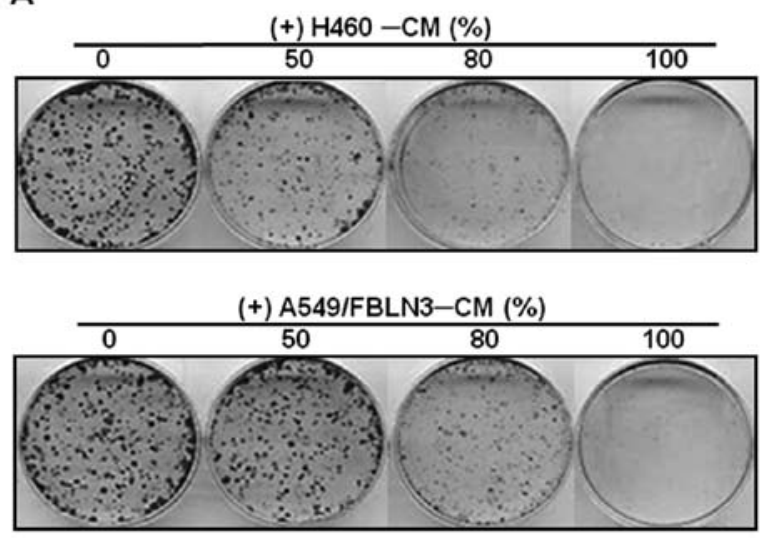

B

C

$(-) \quad(+) \mathrm{H} / \mathrm{C}(+) \mathrm{A} / \mathrm{F} / \mathrm{C}$

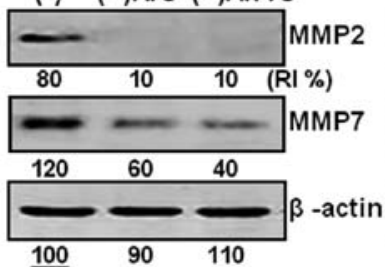

$(-) \quad(+) \mathrm{H} / \mathrm{C}(+) \mathrm{A} / \mathrm{F} / \mathrm{C}$
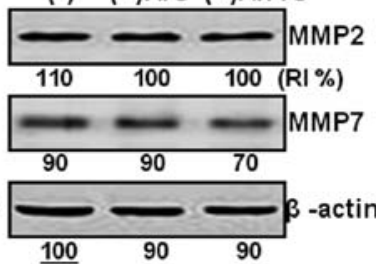

Figure 5. Extracellular fibulin-3 suppressed cell growth and expression of MMPs in A549 cells. (A) Colony-forming assay. A549 cells were plated in 35-mm culture dishes at a density of $2 \times 10^{3}$ cells per plate and incubated. After $24 \mathrm{~h}$, culture media were changed into media made by mixing conditioned media from A549 cells and conditioned media from $\mathrm{H} 460$ cells or fibulin-3-overexpressing A549 cells at indicated concentrations. Cells were then incubated for 10 days and stained with crystal violet. (B) Western blot analysis. A549 cells were plated in $100-\mathrm{mm}$ culture dishes at a density of $5 \times 10^{5}$ cells per plate and incubated After $24 \mathrm{~h}$, culture media were changed into media made by mixing conditioned media from A549 cells and conditioned media from $\mathrm{H} 460$ cells $(\mathrm{H} / \mathrm{C})$ or fibulin3-overexpressing A549 cells $(\mathrm{A} / \mathrm{F} / \mathrm{C})$ at 4:1 ratio. Cells were then incubated for $48 \mathrm{~h}$ and analyzed by Western blotting. (C) To block the function of fibulin-3 in conditioned media, anti-fibulin-3 antibody was added to conditioned media at concentration of $0.4 \mu \mathrm{g} / \mathrm{ml}$ and Western blot analysis was performed as described above.

share a common structure including tandem arrays of calciumbinding EGF domains and a carboxyl-terminal fibulin domain (9). Fibulins seem to be biologically important because mutations in their genes have been associated with several human diseases, such as Malattia Leventinese (26) and cutis laxa (27), though their precise functions and how defects cause disease are not fully understood. Fibulins are also involved in oncogenic and tumour-suppressive roles. Of the seven fibulins, cancerassociated functions have been documented for fibulin-1, -2, -4 and -5 . Expression of fibulin-1 protein is increased in ovarian and breast cancer (28). Fibulin-2 also has been identified as one of 64 overexpressed metastasis-associated genes in solid tumours of diverse types (29). Overexpression of fibulin-4 transforms rat embryonic fibroblasts and increases tumour cell growth in vitro (30). Fibulin-5 overexpression also augments the tumourigenicity of human HT1080 fibrosarcoma cells by increasing their DNA synthesis, migration toward fibronectin and invasion through synthetic basement membranes (11). On the other hand, overexpression of fibulin-1D reduces anchorage-independent growth in vitro and tumour growth in vivo and inhibits cell adhesion, spreading, motility and invasion in vitro (31). In some primary and metastatic tumours, fibulin-5 was reduced (11) and identified as an inhibitor of angiogenesis (13). These show the positive and negative effects of fibulins on cell growth in vitro depending on cell type. Recently, reports about the expression of fibulin-3 in tumour cells have also accumulated. In gliomas and cultured glioma cells, fibulin-3 is highly up-regulated (32). Furthermore, highly expressed fibulin-3 can promote motility and invasiveness of malignant gliomas. Fibulin-3 is also up-regulated in aggressive pancreatic adenocarcinoma, resulting in enhanced in vivo orthotopic and metastatic tumour growth (33). Conversely, fibulin- is down-regulated in primary breast tumours, lung cancer and fibrosarcomas $(6,14,34)$ and is thought to inhibit tumour growth. Fibulin-3 is also down-regulated in nasopharyngeal carcinomas and involved in regulation of invasiveness (35). These results suggest that fibulin-3 has dual functions as a positive and negative regulator of cancer cell growth depending on cell type, although its function as a cell modulator is not clearly understood.

In this study, fibulin-3 expression in NSCLC cells was revealed to be associated with the regulation of tumour cell growth and invasiveness by comparing two lung cancer cell lines. Moreover, the mechanism of fibulin-3 for suppressing invasion of tumour cells was identified as negative regulation of the expression of MMP-7 and MMP-2. Actually, there was evidence of suppression of fibulin-3 expression in lung cancer tissue compared with normal tissue. In a comparison of the expression of six fibulin family genes using NCBI SAGE databases containing about 160,000 transcripts from lung cancer or normal lung tissue, fibulin-3, in addition to fibulin-1, -2 and -5 , was lower in lung cancer (15), which emphasizes the importance of fibulin-3 as a tumour suppressor in lung cancer.

The cell surface target receptor of fibulin-3 in lung cancer cells is not clearly understood at present, although the negative regulation of Erk, Akt and NF- $\kappa \mathrm{B}$ signalling by fibulin-3 was shown in this study. In the case of fibulin-5, it was found that suppression of tumour invasion of lung cancer cells is mediated by the RGD motif of fibulin-5, which targets integrin signalling and consequently inhibits Erk activation and invasion. In the case of fibulin-3, its binding to the EGF receptor had been shown to activate Erk and Akt pathways in pancreatic carcinoma cells (36). However, overexpression of fibulin-3 in A549 lung cancer cells inactivated Erk, Akt and NF- $\kappa \mathrm{B}$ pathways, which excluded EGF-mediated signalling. Moreover, unlike fibulin-5, fibulin-3 does not possess an RGD domain, which confers an action motif to integrin signalling. Therefore, the action of fibulin-3 in NSCLC must be executed by another receptor, which would be investigated in further studies.

Recently, epigenetic modifications associated with cancer have drawn attention, especially promoter hypermethylation leading to transcriptional silencing of tumour suppressor genes. A549 and H460 NSCLC cell lines have similar histologies, but differ greatly in their invasive behaviours and responses to ionizing radiation $(18,19)$. In a previous study, through comparison of mRNA expression profiles of these cell lines and analysis of the methylation pattern of promoter regions, TSPYL5 was identified as a novel oncogenic factor, the expression of which is regulated by promoter hypermethylation (17). We again analyzed mRNA profiles of these NSCLC cell lines to identify 
novel factors involved in their invasive behaviours and identified fibulin-3 as a novel tumour suppressor in NSCLC cells, whose expression is also regulated by promoter methylation.

Taken together, our results suggest that fibulin-3 negatively modulates the invasiveness of lung cancer cells via regulation of MMP-7 and MMP-2 and its expression is regulated by hypermethylation of the promoter region. These facts imply the diagnostic and therapeutic potential of fibulin-3 for aggressive NSCLC and further studies are needed for its clinical applications.

\section{Acknowledgements}

Funding for this study was provided by the Ministry of Education, Science and Technology (Nuclear Research \& Development Program) of the Republic of Korea and the Korea Atomic Energy Research Institute (Basic Research Program) and the Korea Science and Engineering Foundation (KOSEF) grant (OGM2001013).

\section{References}

1. Jones PA and Baylin SB: The fundamental role of epigenetic events in cancer. Nat Rev Genet 3: 3415-3428, 2002.

2. Jones PA and Baylin SB: The epigenomics of cancer. Cell 128: 638-692, 2007.

3. Kim TY, Zhong S, Fields CR, Kim JH and Robertson KD: Epigenomic profiling reveals novel and frequent targets of aberrant DNA methylation-mediated silencing in malignant glioma. Cancer Res 66: 7490-7501, 2006.

4. Alaminos M, Davalos V and Ropero S: EMP3, a myelin-related gene located in the critical 19q13.3 region, is epigenetically silenced and exhibits features of candidate tumor suppressor in glioma and neuroblastoma. Cancer Res 65: 2565-2571, 2005.

5. Shames DS, Minna JD and Gazdar AF: DNA methylation in health, disease, and cancer. Curr Mol Med 7: 85-102, 2007.

6. Yue W, Dacic S, Sun Q, et al: Frequent inactivation of RAMP2, EFEMP1 and Dutt1 in lung cancer by promoter hypermethylation. Clin Cancer Res 13: 4336-4344, 2007.

7. Zhang Y, Wang R, Song H, et al: Methylation of multiple genes as a candidate biomarker in non-small cell lung cancer. Cancer Lett 303: 21-28, 2011.

8. Wang R, Zhang YW and Chen LB: Aberrant promoter methylation of FBLN-3 gene and clinicopathological significance in non-small cell lung carcinoma. Lung Cancer 69: 239-244, 2010.

9. Timpl R, Sasaki T, Kostka G and Ghu MI: Fibulins: versatile family of extracellular matrix proteins. Nat Rev Mol Cell Biol 4 479-489, 2003.

10. De Vega S, Iwamoto T and Yamada Y: Fibulins: multiple roles in matrix structures and tissue functions. Cell Mol Life Sci 66 : 1890-1902. 2009.

11. Schiemann WP, Blobe GC, Kalume DE, Pandey A and Lodish HF: Context-specific effect of fibulin-5(DANCE/EVEC) on proliferation, motility, and invasion: fibulin- 5 is induced by TGF- $\beta$ and affects protein kinase cascades. J Biol Chem 277: 27367-27377, 2002.

12. Albig AR and Schimann WP: Fibulin-5 antagonizes vascular endothelial growth factor signaling and angiogenic sprouting by endothelial cells. DNA Cell Biol 23: 367-379, 2004.

13. Albig AR, Neil JR and Schimann WP: Fibulin-3 and -5 antagonize tumor angiogenesis in vivo. Cancer Res 66: 2621-2629, 2006.

14. Yue W, Sun Q, Landreneau R, Wu C, Siegfried JM, Yu J and Zhang L: Fibulin-5 suppress lung cancer invasion by inhibiting matrix metalloproteinase-7 expression. Cancer Res 69: 6339-6346, 2009.

15. Ghallagher WM, Currid CA and Whelan LC: Fibulins and cancer: friend or foe? Trends Mol Med 11: 336-340, 2005.
16. Tost $\mathrm{J}$ and Gut IG: DNA methylation analysis by pyrosequencing. Nat Protoc 2: 2265-2275, 2007.

17. Kim EJ,Lee SY, Kim TR, Choi SI, Cho EW, Kim KC and Kim IG: TSPYL5 is involved in cell growth and resistance to radiation in A549 cells via the regulation of p21waf1/cip1 and PTEN/Akt pathway. Biochem Biophys Res Commun 392: 448-453, 2010.

18. Das AK, Sato M and Story MD: Non-small-cell lung cancers with kinase domain mutations in the epidermal growth factor receptor are sensitive to ionizing radiation. Cancer Res 66: 9601-9608, 2006.

19. Kraus-Berthier L, Jan M, Guilbaud N, Naze M, Pierré A and Atassi G: Histology and sensitivity to anticancer drugs of two human non-small cell lung carcinomas implanted in the pleural cavity of nude mice. Clin Cancer Res 6: 297-304, 2000.

20. Li LC and Dahiya R: MethPrimer: designing primers for methylation PCRs. Bioinformatics 18: 1427-1431, 2002.

21. Wang YK, Zhu YL, Qiu FM, Zhang T, Chen ZG, Zheng S and Huang J: Activation of Akt and MAPK pathways enhances the tumorigenicity of CD133+ primary colon cancer cells. Carcinogenesis 31: 1376-1380, 2010.

22. Shen X, Artinyan A, Jackson D, Thomas RM, Lowy AM and Kim J: Chemokine receptor CXCR4 enhances proliferation in pancreatic cancer cells through AKT and ERK dependent pathways. Pancreas 39: 81-87, 2010.

23. Zhang Z, Song T, Jin Y, Pan J,Zhang L, Wang L and Li P: Epidermal growth factor receptor regulates MT1-MMP and MMP-2 synthesis in SiHa cells via both PI3-K/AKT and MAPK/ERK pathways. Int J Gynecol Cancer 19: 998-1003, 2009.

24. Wu HH, Cheng YW, Chang JT, Wu TC, Liu WS, Chen CY and Lee H: Subcellular localization of apurinic endonuclease 1 promotes lung tumor aggressiveness via NF-kappaB activation. Oncogene 29: 4330-4340, 2010.

25. Kessenbrock K, Plaks V and Werb Z: Matrix metalloproteinases: regulators of the tumor microenvironment. Cell 141: 52-67, 2010.

26. Stone EM, Lotery AJ, Munier FL, et al: A single EFEMP1 mutation associated with both Malattia Leventinese and Doyne honeycomb retinal dystrophy. Nat Genet 22: 199-202, 1999.

27. Loeys BL, Coucke PJ, De Paepe A, et al: Fibulin-5 mutations: mechanisms of impaired elastic fiber formation in recessive cutis laxa. Hum Mol Genet 15: 3379-3386, 2006.

28. Bardin A,Moll F, Margueron R, et al: Transcriptional and posttranscriptional regulation of fibulin-1 by estrogens leads to differential induction of messenger ribonucleic acid variants in ovarian and breast cancer cells. Endocrinology 146: 760-768, 2005.

29. Argraves WS, Greene LM, Cooley MA and Gallagher WM: Fibulins: physiological and disease perspectives. EMBO Rep 4: 1127-1131, 2003.

30. Sicot FX, Tsuda T, Markova D, et al: Fibulin-2 is dispensable for mouse development and elastic fiber formation. Mol Cell Biol 28: 1061-1067, 2008.

31. Qing J, Maher VM, Tran H, Argraves WS, Dunstan RW and McCormick JJ: Suppression of anchorage-independent growth and matrigel invasion and delayed tumor formation by elevated expression of fibulin-1D in human fibrosarcoma-derived cell lines. Oncogene 15: 2159-2168, 1997.

32. Hu B, Thirtamara-Rajamani KK, Sim H and Viapiano MS: Fibulin-3 is uniquely upregulated in malignant gliomas and promotes tumor cell motility and invasion. Mol Cancer Res 7: 1756-1770, 2009.

33. Seeliger H, Camaj P, Ischenko I, et al: EFEMP1 expression promotes in vivo tumor growth in human pancreatic adenocarcinoma. Mol Cancer Res 7: 189-198, 2009.

34. Sadr-Nabavi A, Ramser J, Volkmann J, et al: Decreased expression of angiogenesis antagonist EFEMP1 in sporadic breast cancer is caused by aberrant promoter methylation and points to an impact of EFEMP1 as molecular biomarker. Int J Cancer 124: 1727-1735, 2009.

35. Hwang CF, Chien CY, Huang SC, et al: Fibulin-3 is associated with tumour progression and a poor prognosis in nasopharyngeal carcinomas and inhibits cell migration and invasion via suppressed AKT activity. J Pathol 222: 367-379, 2010.

36. Camaj P, Seeliger H, Ischenko I, et al: EFEMP1 binds the EGF receptor and activates MAPK and Akt pathways in pancreatic carcinoma cells. Biol Chem 390: 1293-1302, 2009. 